\title{
Independência funcional e comprometimento motor em indivíduos pós-ave da comunidade
}

\section{Functional independence and motor impairment post stroke in individuals in the community}

(D)éssica Rissetti ${ }^{1}$, DJuliana Bertuol Feistauer ${ }^{1}$, DJhoanne Merlyn Luiz ${ }^{1}$, (DLarissa de Souza da Silveira ${ }^{1}$, Angélica Cristiane Ovando ${ }^{1}$

\section{RESUMO}

Indivíduos acometidos por acidente vascular encefálico (AVE) podem apresentar déficits motores, como a hemiparesia, que limitam a autonomia para realização das atividades de vida diária (AVD). Objetivo: Avaliar o comprometimento motor e a independência funcional de indivíduos pósAVE cadastrados nas Unidades Básicas de Saúde (UBS) no município de Araranguá/SC. Método: A amostra foi composta por indivíduos com sequelas motoras de AVE cadastrados nas UBS de Araranguá, que foram avaliados através da Escala de Rankin Modificada, Mini Exame do Estado Mental, Medida de Independência Funcional (MIF), Escala de Fugl Meyer (EFM) e questionário sociodemográfico. Resultados: Foram avaliados 56 indivíduos, com média de idade de $67,3 \pm 11$ anos. Na MIF, 67,9\% apresentaram dependência modificada, necessitando de até $25 \%$ de auxílio para realizar suas AVD. Na pontuação total da EFM, 58,9\% dos indivíduos apresentaram comprometimento marcado, 32,1\% apresentaram comprometimento grave para membro inferior e $46,4 \%$ apresentaram comprometimento moderado para membro superior. Foi observada correlação significativa entre o resultado da EFM total com o resultado da MIF total $(\rho=0.6$; $\rho<0,01)$ e entre o resultado da EFM total com todos os itens da escala MIF $(\rho<0,01)$. Conclusão: Os indivíduos avaliados apresentaram comprometimento motor marcado e algum grau de dependência funcional. A correlação significativa entre as variáveis demonstrou que indivíduos pós-AVE crônico no município de Araranguá apresentam comprometimento motor que gera dependência funcional para realização das AVD. Espera-se que os resultados encontrados possam auxiliar profissionais de saúde da região a criarem abordagens para manter estes indivíduos mais independentes.

Palavras-chave: Acidente Vascular Cerebral, Paresia, Atividades Cotidianas

${ }^{1}$ Universidade Federal de Santa Catarina - UFSC

\section{Correspondência}

Angélica Cristiane Ovando

E-mail: angelica.cristiane@ufsc.br

Submetido: 20 Maio 2020

Aceito: 27 Junho 2020

\section{Como citar}

Rissetti J, Feistauer JB, Luiz JM, Silveira LS, Ovando AC. Independência funcional e comprometimento motor em indivíduos pós-ave da comunidade. Acta Fisiatr. 2020;27(1)27-33

DOI: 10.11606/issn.2317-0190.v27i1a169615

\begin{abstract}
Individuals affected by stroke may have motor deficits, such as hemiparesis, which can limit autonomy to perform activities of daily living (ADLs). Objective: To evaluate the motor impairment and the functional independence of post-stroke individuals registered in Basic Health Units (BHU) in the city of Araranguá/SC. Method: The sample consisted of individuals with sequelae of stroke registered in the Basic Health Units (BHU) of Araranguá, selected in an intentional nonprobabilistic manner. Individuals were assessed using the Modified Rankin Scale, Mini Mental State Examination, Functional Independence Measure (FIM), Fugl Meyer Scale (FMS) and socioeconomic questionnaire. Results: 56 individuals were evaluated, mean age of $67.3 \pm 11$. According to FIM scores, $67.9 \%$ had a modified dependence, requiring up to $25 \%$ assistance to perform their ADLs. In the total FMS score, $58.9 \%$ of the individuals had marked impairment. $32.1 \%$ had severe impairment for the lower limb and $46.4 \%$ had moderate impairment for the upper limb. A significant correlation was observed between the result of the total FMS with the result of the total FIM $(\rho=0.6 ; \rho<0.01)$ and between the result of the total FMS with all the items of the FIM scale $(\rho<0.01)$. Conclusion: The evaluated individuals presented marked motor impairment and some level of functional dependence. The significant correlation between the variables demonstrated that individuals after chronic stroke in Araranguá have motor impairment that generates functional dependence for performing ADLs. It is hoped that the results found will help health professionals in the region to create approaches to keep these individuals more independent.
\end{abstract}

Keywords: Stroke, Paresis, Activities of Daily Living 


\section{INTRODUÇÃO}

O acidente vascular encefálico (AVE) representa a terceira causa de morte mais comum em países ocidentais, sendo a causa mais importante de incapacidade grave em adultos. ${ }^{1,2}$ Os sobreviventes de um AVE, na sua maioria, apresentam deficiências neurológicas e incapacidades residuais significativas. ${ }^{3} \mathrm{O}$ mais importante déficit apresentado pós-AVE é a hemiparesia, que se caracteriza por fraqueza no hemicorpo contralateral à lesão. ${ }^{4}$ Até 85\% dos pacientes apresentam hemiparesia imediatamente após o AVE, e entre 55 a 75\% dos sobreviventes continuam a experimentar déficits motores associados, muitas vezes resultando em prejuízos que podem limitar a autonomia e funcionalidade nas atividades de vida diária (AVD) e instrumentais, afetando diretamente a qualidade de vida e a condição de saúde. ${ }^{5,6}$

O AVE pode causar repercussões sociais e econômicas nos indivíduos, familiares e comunidade. Os acometidos precisam de cuidados substanciais devido às sequelas limitantes, físicas e emocionais, que na maioria dos casos acompanharão o indivíduo para o resto de sua vida. ${ }^{7}$ Espera-se que, aproximadamente, $60 \%$ dos sobreviventes recuperem a independência para 0 autocuidado e $75 \%$ recuperem a marcha independente, sendo que $20 \%$ necessitarão de cuidados institucionais. ${ }^{8}$

$\mathrm{Na}$ população brasileira, mais de $50 \%$ dos sobreviventes de AVE na fase crônica encontram-se limitados para suas AVD. ${ }^{9-12}$ Este percentual de sobreviventes do AVE na fase crônica com limitação funcional é alarmante e merece investigação. Conhecer o perfil funcional de indivíduos acometidos por AVE é importante para os gestores de saúde e fisioterapeutas melhorarem o atendimento à saúde das pessoas pós-AVE e seus familiares. Um estudo recente realizado em Minas Gerais avaliou 44 indivíduos pós AVE que faziam uso do serviço de atenção primária de saúde e identificou incapacidades motoras, funcionais e restrições na participação desta população. ${ }^{3}$

Em Santa Catarina, um estudo realizado em Florianópolis reportou que mais de $50 \%$ dos indivíduos com AVE crônico apresentam sequelas motoras e funcionais, e mesmo os independentes apresentaram restrições na qualidade de vida. ${ }^{13}$

Nada se sabe sobre o perfil funcional dos indivíduos pós-AVE no interior de Santa Catarina, incluindo a cidade de Araranguá e região Sul do estado. A identificação do perfil de saúde e função da população de uma determinada região pode permitir identificar as necessidades comuns desses indivíduos. Isso permite uma melhor orientação dos cuidados oferecidos às pessoas que vivem na mesma área e que apresentam condições semelhantes, e a identificação de possíveis melhorias ou modificações nas estratégias de tratamento para essa população. Para esse fim, são necessários estudos que mostrem resultados além das características das pessoas em uma amostra de conveniência.

Nesse contexto, tem havido grande interesse em informações relacionadas à função e perfil de saúde de indivíduos brasileiros com AVE. Assim, neste estudo, foi avaliada a influência do comprometimento motor na independência funcional de indivíduos pós-AVE cadastrados nas unidades básicas de saúde (UBS) no município de Araranguá/SC. Espera-se que a pesquisa possa proporcionar maior conhecimento a respeito do acometimento desses indivíduos e por meio dela seja possível buscar estratégias de intervenção para minimizar os níveis de incapacidade e dependência.

\section{OBJETIVO}

Este estudo teve como objetivo conhecer as características do comprometimento motor e independência funcional de indivíduos pós-AVE atendidos nas UBS do município de Araranguá-SC.

\section{MÉTODO}

Trata-se de um estudo descritivo e analítico, transversal. Esta pesquisa está de acordo com os princípios éticos contidos nas Resoluções no 466/2012 e no 510/2016, tendo sido aprovada pelo Comitê de Ética em Pesquisa da Universidade Federal de Santa Catarina (UFSC) sob o número de CAAE: 67843317.3.0000.0121. Todos os participantes do estudo assinaram o Termo de Consentimento Livre e Esclarecido (TCLE).

A amostra foi composta por indivíduos com sequelas pós-AVE cadastrados nas UBS do município de Araranguá, com diagnóstico de AVE no prontuário, selecionados de maneira não probabilística intencional, correspondendo aos critérios de inclusão e que aceitaram participar do estudo.

Foram incluídos nesse estudo indivíduos adultos (mais de 18 anos), de ambos os sexos, que apresentaram sequelas motoras de AVE crônico (mais de seis meses), residentes do município de Araranguá/SC, que aceitaram participar da pesquisa e apresentaram escore na Escala de Rankin Modificada (ERM) a partir do nível 2.

Foram excluídos indivíduos que apresentaram AVE em menos de seis meses, aqueles que não apresentaram nenhuma sequela ou aqueles que permaneceram apenas com sequelas cognitivas (sem sequelas motoras), indivíduos com alguma outra doença incapacitante associada e aqueles que não apresentaram capacidade cognitiva suficiente para responder às avaliações, de acordo com o Mini Exame do Estado Mental (MEEM), com pontos de corte de acordo com Bertolucci et al. ${ }^{14}$

Foram realizadas análises de todos os prontuários físicos de cada uma das 15 UBS do município, sendo então registrado o número de indivíduos encontrados com o diagnóstico médico de AVE. Foram coletadas informações referentes ao nome, data de nascimento, telefone, endereço, número de prontuário e número do cartão família. Após a análise dos prontuários, foi realizada tentativa de contato com todos os indivíduos encontrados na triagem que apresentaram número de telefone ou endereço, e verificado se os mesmos se enquadravam aos critérios de inclusão para então convidá-los a participar do estudo. Neste contato, foram expostos os objetivos do estudo, a importância da participação, os instrumentos utilizados, o sigilo das informações e o convite para participar das avaliações.

Após o aceite do indivíduo que se enquadrava nos critérios, uma visita à residência do mesmo foi marcada. Após a assinatura do TCLE um fisioterapeuta realizou a avaliação do indivíduo com a aplicação dos instrumentos de pesquisa.

A Escala de Rankin Modificada (ERM) foi utilizada para a mensuração da incapacidade dos indivíduos pós-AVE. Essa escala apresenta seis categorias que vão do 0 (sem sintomas) a 5 (deficiência grave), agregando-se, eventualmente, o escore 6 (óbito). É um método sistemático de avaliar a capacidade do paciente, identificando habilidades ou deficiências no autocuidado e as necessidades relacionadas às atividades diárias. ${ }^{15}$

A Medida de Independência Funcional (MIF) foi utilizada para mensuração da independência funcional dos indivíduos pósAVE na realização das suas atividades de vida diária. $\mathrm{O}$ instrumento foi elaborado para mensurar a capacidade funcional por meio de uma escala de sete níveis que representam os graus de funcionalidade, variando da independência à dependência. A classificação de uma atividade em termos de dependência 
ou independência é baseada na necessidade de ser assistido ou não por outra pessoa e, se a ajuda é necessária, em qual proporção. ${ }^{16}$

A MIF completa pode ser dividida em quatro sub escores, segundo a pontuação total obtida: a) 18 pontos: dependência completa (assistência total); b) 19 a 60 pontos: dependência modificada (assistência de até $50 \%$ das tarefas); c) 61 a 103 pontos: dependência modificada (assistência de até 25\% das tarefas); d) 104 a 126 pontos: independência completa. ${ }^{17}$ Assim, quanto menor a pontuação, maior será o grau de dependência do avaliado. A partir da soma dos pontos obtidos em cada dimensão, temse um escore mínimo de 18 e máximo de 126 pontos, que vão caracterizar os níveis de dependência. ${ }^{16} \mathrm{O}$ instrumento foi aplicado por profissional devidamente treinado para tal.

A Escala de Fugl-Meyer (EFM) foi utilizada para mensuração do comprometimento motor pós-AVE. Essa escala apresenta quatro domínios: amplitude de movimento passiva e dor, sensibilidade, função motora da extremidade superior e inferior e equilíbrio, além da coordenação e velocidade. Para o presente estudo foram utilizados somente os itens da função motora da extremidade superior e inferior. $O$ escore total da função motora normal é de 100 pontos, em que a pontuação máxima para a extremidade superior é 66 e para a inferior 34. ${ }^{7,18}$

Fugl-Meyer et al. determinaram uma pontuação de acordo com o nível de comprometimento motor, em que menos que 50 pontos indicam um comprometimento motor severo; 50-84 marcante; 85-95 moderado; e $96-99$ leve. ${ }^{19}$

Além dos instrumentos, foram coletadas informações sociodemográficas dos indivíduos como nome, sexo, data de nascimento, estado civil, escolaridade, tipo e tempo do $A V E$, se realizou fisioterapia após o episódio e por quanto tempo, se apresentava outras doenças associadas, quais os medicamentos utilizados, além de informações sobre renda. Essas informações foram utilizadas para caracterizar o perfil do participante.

As variáveis do estudo foram analisadas descritivamente por meio de frequências absolutas e relativas e medidas de tendência central e dispersão. Coeficientes de correlação de Spearman foram calculados para avaliar a magnitude, direção e significância das correlações entre as variáveis: independência funcional e comprometimento motor gerada pelo AVE. Nas análises inferenciais descritas foi considerado um nível de significância de $p<0,05$, utilizando o pacote estatístico SPSS versão 20.0 para Windows.

\section{RESULTADOS}

A seguir são apresentados os resultados da triagem dos indivíduos do estudo (Figura 1). De 564 indivíduos encontrados após as análises de todos os prontuários físicos das 15 UBS, 56 indivíduos corresponderam aos critérios de inclusão e aceitaram participar do estudo, compondo a amostra final.

A Tabela 1 apresenta as características dos indivíduos avaliados. Observa-se que a maioria dos participantes eram do sexo masculino, sendo o lado esquerdo o mais acometido, com predomínio de AVE isquêmico. Além disso, a maioria dos indivíduos era casado e a média de idade foi $67,3 \pm 11$ anos. Ainda, a maioria dos indivíduos apresentou em média duas doenças associadas, como mostrado na Tabela 1, sendo as doenças mais prevalentes a hipertensão arterial (93\% dos pacientes) e diabetes (75\% dos pacientes) .

Em relação à avaliação da dependência para as AVD, avaliada pela MIF, foi observado que dos 56 indivíduos avaliados, nenhum deles apresentou dependência completa e 38 apresentaram dependência modificada, necessitando de assistência de até $25 \%$ na realização de suas atividades de vida diárias.

Ainda, 16 indivíduos apresentaram independência completa e apenas dois apresentaram dependência modificada, necessitando de assistência para até $50 \%$ da realização de suas AVDs.

$\mathrm{Na}$ avaliação dos itens e domínios individuais da MIF (Tabela 2), foi observado que no domínio motor, o item escadas foi o que obteve menor pontuação, com média de 3,2 pontos. Já no domínio cognitivo, o item que obteve menor média foi resolução de problemas com média de 4,7 pontos.

A Tabela 3 apresenta os resultados do comprometimento motor de membros superiores e inferiores do indivíduos acometidos por AVE, avaliado pela EFM e a Tabela 4 apresenta o resultado da correlação entre o comprometimento motor, avaliado pela EFM e os itens da MIF.

\section{DISCUSSÃO}

Este estudo avaliou o comprometimento motor e a independência funcional de indivíduos pós-AVE cadastrados nas UBS no município de Araranguá-SC. Com relação às características da amostra, foi observado que a maioria dos indivíduos inseridos no estudo são do sexo masculino, idosos, em sua maioria acometidos por AVE isquêmico, com o lado esquerdo predominantemente acometido, tendo realizado fisioterapia após o episódio de AVE e apresentando principalmente como doenças associadas, diabetes e hipertensão.

Em todo o mundo, o AVE é mais comum entre os homens, embora as mulheres permanecem com sequelas mais acentuadas. ${ }^{20}$ A idade é um importante fator para a incidência do $A V E$, já que o risco aumenta duas vezes a cada dez anos depois dos 55 anos de idade. ${ }^{17,20}$ No que se refere ao hemicorpo mais acometido, a literatura mostra variações, sendo que alguns autores ${ }^{18,21}$ relataram a ausência de diferença entre o grau de independência funcional de indivíduos com AVE à esquerda ou à direita.

Já no estudo de Voos e Ribeiro, ${ }^{22}$ foi observado que em relação à independência funcional, o desempenho inicial mostrou-se mais comprometido no grupo com lesão à esquerda do que no grupo com lesão à direita, mas a taxa de recuperação funcional não diferiu significativamente entre eles.

Em relação aos resultados do comprometimento motor avaliado pela EFM, foi observado que $46,4 \%$ dos indivíduos apresentaram comprometimento moderado no membro superior e $32,1 \%$ apresentaram comprometimento grave em membro inferior.

No resultado total da EFM, 58,9\% apresentaram comprometimento marcado. Resultados semelhantes são observados em outro estudo ${ }^{13}$ que avaliou o grau de recuperação motora, independência funcional e qualidade de vida em 130 indivíduos pósAVE crônico, em Florianópolis - SC, e observou que na subescala de membro superior da EFM a maioria da amostra distribuiu-se em comprometimento moderado $(21,5 \%)$ a severo $(31,5 \%)$, demonstrando comprometimento residual em mais da metade dos participantes e na subescala de membro inferior, os autores também encontraram comprometimento motor marcado a grave em $31,5 \%$.

No estudo de Teles e Gusmão, ${ }^{23}$ a função do membro inferior mostrou-se menos comprometida em relação ao superior, e apesar das funções de todos os indivíduos estarem comprometidas, em nenhum a função apresentou-se ausente. Já em outro estudo, ${ }^{24}$ foram demonstrados resultados semelhantes entre o comprometimento motor das extremidades superior e inferior. Neste estudo, a relação entre a recuperação motora da extremidade superior e inferior apresentou algumas disparidades, porém em nenhum a função esteve ausente. 


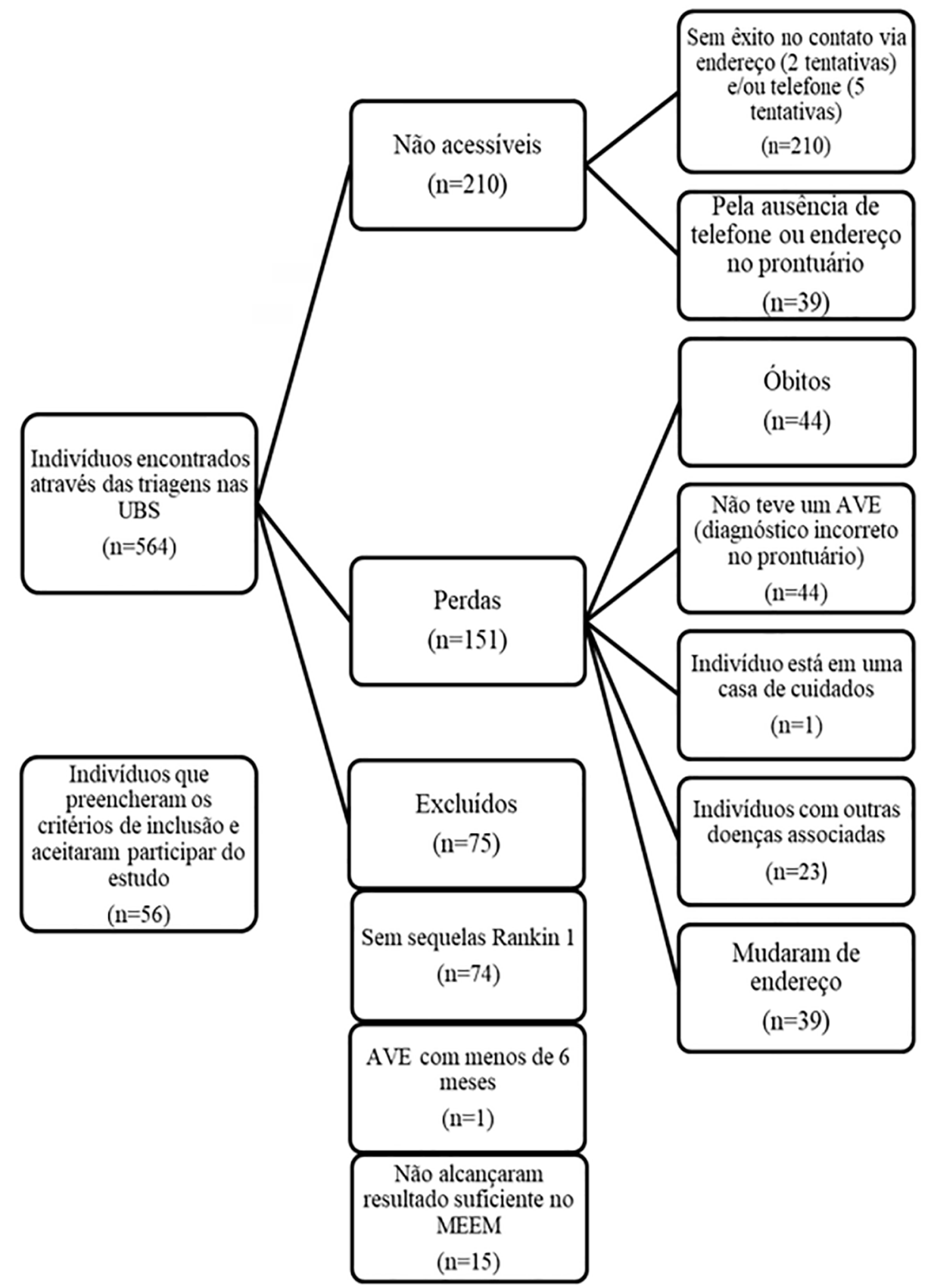

Figura 1. Fluxograma de recrutamento do estudo

Dados semelhantes foram encontrados no estudo de Viana et al. ${ }^{12}$ que demonstrou que os idosos com sequelas de AVE apresentam dificuldade em mais de $75 \%$ das vezes para resolver problemas como: verificar uma conta, autoadministrar medicamentos, confrontar problemas interpessoais e lidar com as situações não planejadas ou ocasionais, como pedir auxílio apropriado durante uma transferência, desabotoar uma camisa após tentar vesti-la, dentre outras. Pode-se dizer que este resultado para o domínio cognitivo já era esperado, tendo em vista que a população foi selecionada através da avaliação do Mini Exame do Estado Mental, sendo excluídos do estudo aqueles indivíduos que apresentassem comprometimento cognitivo que os impedisse de responder aos questionários de avaliação.

Com relação ao domínio motor da MIF, foi observado que o item controle de esfíncteres (fezes) foi o que obteve maior pontuação, com média de 6 pontos, indicando dependência modificada com necessidade apenas de dispositivos auxiliares; seguido por controle de esfíncteres (urina), alimentação e higiene pessoal, indicando necessidade de supervisão ou preparo. $O$ controle esfincteriano é referido como estando fortemente relacionado a um melhor prognóstico, motor e cognitivo, em pacientes pós-AVE. ${ }^{16}$

Já os itens banho e vestir a metade superior e inferior obtiveram escores com média abaixo de 5 pontos, indicando necessidade de assistência com contato mínimo, resultados que vão ao encontro de outros estudos. ${ }^{13,26}$ Vestir-se é uma atividade que demanda várias habilidades como destreza dos membros superiores, equilíbrio, força, amplitude dos membros inferiores, entre outras, ${ }^{18}$ fato este que pode ter contribuído para o nível de dependência encontrado no presente estudo.

A dificuldade na realização desta tarefa já foi observada em estudos prévios, ${ }^{26,27}$ e se relaciona com a deficiência motora do membro superior, comprovado no presente estudo pela correlação significativa da EFM com o item vestir-se da MIF e também apontada por Teles e Gusmão, ${ }^{23}$ que destacam que os movimentos de flexão, abdução de ombro e supinação do braço costumam ser os mais limitados.

Cacho, Melo, Oliveira, ${ }^{24}$ avaliando a recuperação motora de pacientes com hemiplegia através da EFM observaram que após seis meses de tratamento, $20 \%$ dos pacientes ainda apresentavam limitação de amplitude articular e dor, principalmente na flexão, rotação externa e abdução de ombro, seguida de extensão de punhos e dedos. Os autores atribuem esse comprometimento à presença de dores no ombro, como principal causa para este fato.

Ainda no domínio motor, o item escadas demonstrou menor pontuação com média de 3,2 pontos, indicando necessidade de assistência moderada, assim como em outros estudos prévios em que os indivíduos avaliados demonstraram déficit para realização desta atividade., ${ }^{9,13,26}$ Este resultado pode estar relacionado ao fato de que esta é uma atividade com um alto nível de dificuldade de realização para os indivíduos pós-AVE. ${ }^{27}$

O comprometimento grave em membro inferior observado em nosso estudo é um fator relacionado a essa dificuldade, conforme observado na correlação significativa entre o resultado da Escala de Fugl-Meyer e a atividade de subir escadas da MIF. Além disso, a maior demanda física que a escada exige, ${ }^{28}$ 
Tabela 1. Características dos indivíduos avaliados

\begin{tabular}{|c|c|c|c|}
\hline \multirow{2}{*}{ VARIÁVEL } & \multirow{2}{*}{ CATEGORIA } & \multicolumn{2}{|c|}{ RESULTADO } \\
\hline & & $\mathrm{N}$ & $\%$ \\
\hline \multirow{2}{*}{ Sexo } & Masculino & 32 & 57.1 \\
\hline & Feminino & 24 & 42.9 \\
\hline \multirow{3}{*}{ Tipo de AVE } & Isquêmico & 40 & 71.4 \\
\hline & Hemorrágico & 9 & 16 \\
\hline & Não sabe & 7 & 12.5 \\
\hline \multirow{2}{*}{ Lado acometido } & Direito & 26 & 46.4 \\
\hline & Esquerdo & 30 & 53.6 \\
\hline \multirow{4}{*}{ Escolaridade } & Fundamental Incompleto & 48 & 85.7 \\
\hline & Fundamental Completo & 2 & 3.5 \\
\hline & Médio Completo & 5 & 9 \\
\hline & Superior Incompleto & 1 & 1.8 \\
\hline \multirow{4}{*}{ Estado civil } & Casado & 33 & 58.9 \\
\hline & Viúvo & 12 & 21.4 \\
\hline & Divorciado & 4 & 7.1 \\
\hline & Outro & 7 & 12.5 \\
\hline \multirow{3}{*}{ Fez Fisioterapia } & Sim & 43 & 76,8 \\
\hline & Não & 10 & 17,9 \\
\hline & Não informado & 3 & 5,4 \\
\hline Idade & Anos & \multicolumn{2}{|c|}{$67,3 \pm 11$} \\
\hline Tempo de AVE & Anos & \multicolumn{2}{|c|}{$7,6 \pm 7,5$} \\
\hline Doenças associadas & Número de doenças & \multicolumn{2}{|c|}{$2 \pm 1,4$} \\
\hline Medicamentos & Número & \multicolumn{2}{|c|}{$4,6 \pm 2,2$} \\
\hline Renda & Salário mínimo & \multicolumn{2}{|c|}{$2 \pm 0,68$} \\
\hline
\end{tabular}

indica que a aptidão física e a tarefa de subir e descer escadas em indivíduos na fase crônica do AVE precisa ser melhor abordada no processo de reabilitação. ${ }^{13}$

A maioria dos indivíduos da amostra $(76,8 \%)$ relata ter realizado fisioterapia após sofrerem o AVE, o que pode ter sido um fator que tenha de alguma forma influenciado no desempenho das AVD, conforme observado pelo nível de dependência modificada segundo a escala MIF, onde a maioria dos indivíduos necessita de menor assistência durante suas AVD. Sabe-se que a realização da fisioterapia é um componente importante para a manutenção e a melhora do estado funcional e para a prevenção das incapacidades secundárias. ${ }^{29-31}$

Apesar disso, foi observado um alto nível de comprometimento motor nos indivíduos, e isso pode estar ligado ao fato de não terem sido investigadas variáveis relacionadas à realização da fisioterapia (quanto tempo após o AVE procurou reabilitação, por quanto tempo realizou, se foi apenas fisioterapia hospitalar). Além disso, os indivíduos da amostra mostraram baixa renda e nível de escolaridade, o que pode dificultar o acesso à informação, levando a menores rendimentos socioeconômicos, menos conhecimento sobre os fatores de risco para a saúde, prevenção da doença e seu tratamento adequado. ${ }^{32}$

Paolucci et al..$^{33}$ teve como objetivo em seu estudo avaliar a estabilidade do nível de mobilidade alcançado durante a reabilitação hospitalar em indivíduos pós-AVE, e ao longo de 1 ano identificar os fatores associados com as mudanças nestes níveis, e observou que a maioria dos indivíduos apresentaram declínio da mobilidade no segmento de um ano, demonstrando que a reabilitação exerce papel crucial na vida dos indivíduos pós-AVE. Por fim, os autores concluíram que o nível de mobilidade não está estabilizado na alta hospitalar e que a reabilitação pós-alta pode prevenir e aumentar os níveis de mobilidade alcançados durante a internação.

Com isso, os programas de reabilitação devem ser adaptados às necessidades específicas de cada paciente para aumentar o número de indivíduos que aderem aos mesmos e assim reduzir a frequência de institucionalizações.
Tabela 2. Resultado por itens e domínios da Medida de Independência Funcional

\begin{tabular}{|c|c|c|}
\hline Autocuidados & Média & DP \\
\hline Alimentação & 5,3 & 1 \\
\hline Higiene pessoal & 5,2 & 1,4 \\
\hline Banho & 4,9 & 1,7 \\
\hline Vestir-se acima/cintura & 4,7 & 1,8 \\
\hline Vestir-se abaixo/cintura & 4,6 & 1,8 \\
\hline Uso do vaso sanitário & 5,3 & 1,6 \\
\hline \multicolumn{3}{|l|}{ Controle de Esfíncteres } \\
\hline Urina & 5,6 & 1,8 \\
\hline Fezes & 6 & 1,5 \\
\hline \multicolumn{3}{|l|}{ Transferências } \\
\hline Cama/cadeira/CR & 5,5 & 1,1 \\
\hline Vaso sanitário & 5,5 & 1,1 \\
\hline Banheira/chuveiro & 5,2 & 1,4 \\
\hline \multicolumn{3}{|l|}{ Locomoção } \\
\hline Marcha & 5,2 & 1,2 \\
\hline Escadas & 3,2 & 2 \\
\hline Total Motor & 66,2 & 13,9 \\
\hline \multicolumn{3}{|l|}{ Comunicação } \\
\hline Expressão & 6,5 & 0,9 \\
\hline Compreensão & 6,4 & 0,9 \\
\hline \multicolumn{3}{|l|}{ Cognição Social } \\
\hline Interação Social & 6,3 & 0,8 \\
\hline Resolução de Problemas & 4,7 & 1,9 \\
\hline Memória & 5,1 & 1,4 \\
\hline Total Cognitiva & 26,9 & 5,6 \\
\hline MIF TOTAL & 92,9 & 16,5 \\
\hline
\end{tabular}

No presente estudo, foi observada correlação significativa entre o comprometimento motor e a independência funcional em todas as atividades do domínio motor da MIF. Esses resultados que vão ao encontro com a literatura, ${ }^{9,13,26}$ que ao analisarem o comprometimento motor de indivíduos pós-AVE crônico, também concluíram que este impacta em suas AVD's, limitando a dependência funcional dos indivíduos.

A independência para as atividades da vida diária inclui um desempenho satisfatório de diversos movimentos, tais como: levantarse de uma cadeira, flexionar-se e deambular. Para isso o indivíduo precisa ter o domínio do controle postural que é solicitado para a execução dessas atividades, através da capacidade de manter-se em diversas posições, responder automaticamente a movimentos voluntários do corpo e das suas extremidades e reagir adequadamente a perturbações externas. ${ }^{34}$ 
Tabela 3. Resultado da Escala de Fugl-Meyer por segmentos e Fugl-Meyer total

\begin{tabular}{|c|c|c|c|c|}
\hline & $\mathbf{N}$ & $\%$ & Média & DP \\
\hline \multicolumn{5}{|l|}{ EFM membro superior $(0-66)$} \\
\hline Sem Comprometimento $=66$ & 1 & 1,8 & 1,2 & - \\
\hline Leve $(53-65)$ & 10 & 17,9 & 10,2 & 4,3 \\
\hline Moderado (31-52) & 26 & 46,4 & 20,3 & 5,3 \\
\hline Severo $(\leq 30)$ & 19 & 33,9 & 5,9 & 8,3 \\
\hline \multicolumn{5}{|l|}{ EFM membro inferior $(0$ - 34) } \\
\hline Sem Comprometimento $=34$ & 1 & 1,8 & 0,6 & - \\
\hline Leve $(29-33)$ & 5 & 8,9 & 1,6 & 1,4 \\
\hline Moderado (23 - 28) & 17 & 30,4 & 7,6 & 1,8 \\
\hline Marcado (18-22) & 15 & 26,8 & 5,4 & 1,4 \\
\hline Grave ( $\leq 17)$ & 18 & 32,1 & 4,4 & 3,4 \\
\hline \multicolumn{5}{|l|}{ EFM total $(0-100)$} \\
\hline Sem comprometimento $(=100)$ & - & - & - & - \\
\hline Leve (96-99) & 1 & 1,8 & 1,7 & - \\
\hline Moderado (85-95) & 2 & 3,6 & 3,1 & 2,1 \\
\hline Marcado (51-84) & 33 & 58,9 & 39,9 & 8,4 \\
\hline Grave (<50) & 20 & 35,7 & 12,4 & 11,5 \\
\hline
\end{tabular}

$\mathrm{MIF}=$ Medida de Independência Funcional; ${ }^{*}=p<0,01$

Tabela 4. Correlação entre os Itens da MIF e a Escala de Fulg-Meyer

\begin{tabular}{lc}
\hline Item da MIF & Valor da correlação \\
\hline MIF alimentação & $\rho=0,54^{*}$ \\
\hline MIF higiene pessoal & $\rho=0,48^{*}$ \\
\hline MIF banho & $\rho=0,6^{*}$ \\
\hline MIF vestir metade superior & $\rho=0,57^{*}$ \\
\hline MIF vestir metade inferior & $\rho=0.65^{*}$ \\
\hline MIF utilização do vaso sanitário & $\rho=0,37^{*}$ \\
\hline MIF transferência chuveiro & $\rho=0,66^{*}$ \\
\hline MIF marcha/cadeira de rodas & $\rho=0,54^{*}$ \\
MIF escadas & $\rho=0,6^{*}$ \\
\hline MIF total & $\rho=0,6^{*}$ \\
\hline MIF= Medida de Independência Funcional; ${ }^{*}=p<0,01$
\end{tabular}

Os sobreviventes de AVE muitas vezes realizam tarefas através de estratégias de movimentos compensatórios e assim conseguem realizar algumas de suas AVD. Assim, os resultados deste estudo confirmam achados da literatura que demonstram a influência do comprometimento motor prejudicado na realização das AVD's. ${ }^{9,13,25,26}$

Como limitação do presente estudo destacamos o número de indivíduos avaliados, que pode ser considerado um número pequeno já que nosso intuito era atingir toda a população da cidade que já foi acometida por AVE e que apresentava alguma incapacidade. Isso se deve às inúmeras exclusões de indivíduos que não se enquadraram nos critérios do estudo, e também pela dificuldade encontrada durante a triagem, seja pela falta de êxito no contato via endereço, pela ausência de telefone ou endereço no prontuário da UBS e também pelo número de óbitos.

Podemos considerar como diferencial deste estudo o fato de que foi o primeiro a avaliar esta população, suas características e limitações, na cidade de Araranguá. Ainda assim, foram encontrados resultados importantes que demonstram que o grau de comprometimento motor, neste caso, predominantemente grave para membros inferiores dos indivíduos, afeta de forma significativa atividades que envolvam estas extremidades, resultando em uma redução da independência funcional.

\section{CONCLUSÃO}

Os indivíduos avaliados no estudo apresentaram, em sua maioria, comprometimento motor marcado e algum grau de dependência funcional para realização de suas AVD. Houve correlação significativa entre o comprometimento motor e a independência funcional, o que demonstrou que indivíduos pós-AVE crônico no município de Araranguá apresentam comprometimento motor que gera dependência funcional para realização das AVD.
Nossos achados são importantes, pois ajudam a conhecer esta população e podem auxiliar profissionais da área da saúde a criarem abordagens que acompanham pessoas acometidas pelo AVE de forma contínua, proporcionando maior acesso à informação e intervenções visando sua independência funcional.

\section{REFERÊNCIAS}

1. Johnson W, Onuma O, Owolabi M, Sachdev S. Stroke: a global response is needed. Bull World Health Organ. 2016;94(9):634-634A. Doi: http://dx.doi. org/10.2471/BLT.16.181636

2. Feigin VL, Forouzanfar $M H$, Krishnamurthi $R$, Mensah GA, Connor M, Bennett DA, et al. Global and regional burden of stroke during 1990-2010: findings from the Global Burden of Disease Study 2010. Lancet. 2014;383(9913):245-54. Doi: http://dx.doi. org/10.1016/s0140-6736(13)61953-4

3. Carvalho-Pinto BP, Faria CD. Health, function and disability in stroke patients in the community. Braz J Phys Ther. 2016;20(4):355-66. Doi: http://dx.doi. org/10.1590/bjpt-rbf.2014.0171

4. Bohannon RW. Muscle strength and muscle training after stroke. J Rehabil Med. 2007;39(1):14-20. Doi: http://dx.doi.org/10.2340/16501977-0018

5. Carvalho JC, Gusmão CA, Matos MA, Matias AC, Santos NA. Avaliação dos desfechos de funcionalidade e mobilidade pós-acidente vascular encefálico. Rev Fac Ciênc Méd Sorocaba. 2013;15(4):100-4.

6. Yoshida HM, Barreira J, Fernandes PT. Habilidade motora, sintomas depressivos e função cognitiva em pacientes pós-AVC. Fisioter Pesqui. 2019;26(1):9-14. Doi: https://doi.org/10.1590/18092950/17001026012019

7. Lopes Junior JEG, Freitas Junior JHA, Figueiredo ADJ, Santana FM. Perfil dos pacientes acometidos por acidente vascular encefálico cadastrados na estratégia de saúde da família. Rev Fisioter S Func. 2013;2(1):21-7.

8. Terranova TT, Albieri FO, Almeida MD, Ayres DVM, Cruz SF, Milazzotto MV, et al. Acidente vascular cerebral crônico: reabilitação. Acta Fisiatr. 2012;19(2):50-9. Doi: https://doi.org/10.5935/01047795.20120011

9. Fernandes MB, Cabral DL, Souza RJP, Sekitani HY, Teixeira-Salmela LF, Laurentino GEC. Independência funcional de indivíduos hemiparéticos crônicos e sua relação com a fisioterapia. Fisioter Mov. 2012;25(2):333-41. Doi: https://doi.org/10.1590/ S0103-51502012000200011

10. Hartman-Maeir A, Soroker N, Ring H, Avni N, Katz N. Activities, participation and satisfaction one-year post stroke. Disabil Rehabil. 2007;29(7):559-66. Doi: https://doi.org/10.1080/09638280600924996

11. Riberto M, Miyasaki MH, Jucá SSH, Lourenço $C$, Battistella LR. Independência funcional em pessoas com lesões encefálicas adquiridas sob reabilitação ambulatorial. Acta fisiátr. 2007;14(2):87-94.

12. Viana FP, Lorenzo AC, Oliveira EF, Resende SM. Medida de independência funcional nas atividades de vida diária em idosos com sequelas de acidente vascular encefálico no Complexo Gerontológico Sagrada Família de Goiânia. Rev Bras Geriatr Gerontol. 2008;11(1):1728. Doi: https://doi.org/10.1590/18099823.2008.11013 
13. Costa A. Análise do perfil funcional e dos preditores da qualidade de vida relacionada à saúde em indivíduos pós acidente vascular encefálico [Dissertação]. Florianópolis: Universidade do Estado de Santa Catarina; 2016.

14. Bertolucci PH, Brucki SM, Campacci SR, Juliano Y. O mini-exame do estado mental em uma população geral: impacto da escolaridade. Arq Neuropsiquiatr. 1994;52(1):1-7. Doi: https://doi.org/10.1590/S0004282X1994000100001

15. Cincura C, Pontes-Neto OM, Neville IS, Mendes HF, Menezes DF, Mariano DC, et al. Validation of the National Institutes of Health Stroke Scale, modified Rankin Scale and Barthel Index in Brazil: the role of cultural adaptation and structured interviewing. Cerebrovasc Dis. 2009;27(2):119-22. Doi: https://doi. org/10.1159/000177918

16. Riberto $\mathrm{M}$, Miyazaki $\mathrm{MH}$, Jorge Filho $\mathrm{D}$, Sakamoto H, Battistella LR. Reprodutibilidade da versão brasileira da Medida de Independência Funcional. Acta Fisiatr. 2001;8(1):45-52. Doi: https://doi. org/10.5935/0104-7795.20010002

17. Melgaard L, Rasmussen LH, Skjøth F, Lip GY, Larsen TB. Age dependence of risk factors for stroke and death in young patients with atrial fibrillation: a nationwide study. Stroke. 2014;45(5):1331-7. Doi: https://doi.org/10.1161/STROKEAHA.114.004903

18. Laufer Y, Sivan D, Schwarzmann R, Sprecher E. Standing balance and functional recovery of patients with right and left hemiparesis in the early stages of rehabilitation. Neurorehabil Neural Repair. 2003;17(4):207-13. Doi: https://doi.org/10.1177/0888439003259169

19. Michaelsen SM, Rocha AS, Knabben RJ, Rodrigues LP, Fernandes CGC. Tradução, adaptação e confiabilidade interexaminadores do manual de administração da escala de Fugl-Meyer. Rev Bras Fisioter. 2011;15(1):80-8. Doi: https://doi.org/10.1590/\$1413-35552011000100013
20. Barker-Collo $S$, Bennett DA, Krishnamurth RV, Parmar P, Feigin VL, Naghavi M, et al. Sex Differences in stroke incidence, prevalence, mortality and disability-adjusted life years: results from the Global Burden of Disease Study 2013. Neuroepidemiology. 2015;45(3):203-14. Doi: https://doi.org/10.1159/000441103

21. Lucke-Wold BP, Turner RC, Lucke-Wold AN, Rosen $\mathrm{CL}$, Huber JD. Age and the metabolic syndrome as risk factors for ischemic stroke: improving preclinical models of ischemic stroke. Yale J Biol Med. 2012;85(4):523-39.

22. Voos MC, Ribeiro LEV. Estudo comparativo entre a relação do hemisfério acometido no acidente vascular encefálico e a evolução funcional em indivíduos destros. Rev Bras Fisioter. 2008;12(2):113-20. Doi: https://doi. org/10.1590/S1413-35552008000200007

23. Teles MS, Gusmão C. Avaliação funcional de pacientes com acidente vascular encefálico utilizando o protocolo de Fugl-Meyer. Rev Neurocienc. 2012;20(1):42-9. Doi: https://doi.org/10.34024/ rnc.2012.v20.8300

24. Cacho EWA, Melo FRLV, Oliveira R. Avaliação da recuperação motora de pacientes hemiplégicos através do protocolo de desempenho físico Fugl-Meyer. Rev Neurociências. 2004;12(2):94101. Doi: https://doi.org/10.34024/rnc.2004 v12.8877

25. Ricci NA, Kubota MT, Cordeiro RC. Concordância de observações sobre a capacidade funcional de idosos em assistência domiciliar. Rev Saúde Pública. 2005;39(54):655-62. Doi: https://doi org/10.1590/S0034-89102005000400021

26. Riberto $M$, Miyasaki $\mathrm{MH}$, Jucá SSH, Lourenço $C$ Battistella LR. Independência funcional em pessoas com lesões encefálicas adquiridas sob reabilitação ambulatorial. Acta Fisiátr. 2007;14(2):87-94.
27. Amaral-Natalio $M$, Nunes GS, Herber V, Michaelsen SM Relação entre cadência da subida e descida de escada, recuperação motora e equilíbrio em indivíduos com hemiparesia. Acta Fisiátr. 2011;18(3):146-50.

28. Reeves ND, Spanjaard $M$, Mohagheghi AA, Baltzopoulos V, Maganaris CN. Older adults employ alternative strategies to operate within their maximum capabilities when ascending stairs. J Electromyogr Kinesiol. 2009;19(2):e57-68. Doi https://doi.org/10.1016/j.jelekin.2007.09.009

29. Langhorne $P$, Bernhardt J, Kwakkel G. Stroke rehabilitation. Lancet. 2011;377(9778):1693-702. Doi https://doi.org/10.1016/S0140-6736(11)60325-5

30. Lucena EMF, Morais JD, Batista HRL, Mendes LM Silva KSQR, Neves RF, et al. A funcionalidade de usuários acometidos por AVE em conformidade com a acessibilidade à reabilitação. Acta Fisiátr. 2011;18(3):12-8.

31. Cecatto RB, Almeida $\mathrm{Cl}$. O planejamento da reabilitação na fase aguda após o acidente vascular encefálico. Acta Fisiátr. 2010;17(1):37-43.

32. Boaventura $\mathrm{CL}$, Borges $\mathrm{CH}$, Ozaki HA. Avaliação da sobrecarga do cuidador de pacientes neurológicos cadeirantes adultos. Ciênc Saúde Coletiva. 2016;21(10):3193-3202. Doi: https://doi. org/10.1590/1413-812320152110.15202016

33. Paolucci S, Grasso MG, Antonucci G, Bragoni M, Trois $E$, Morelli $D$, et al. Mobility status after inpatient stroke rehabilitation: 1-year follow-up and prognostic factors. Arch Phys Med Rehabil. 2001;82(1):2-8. Doi: https://doi.org/10.1053/apmr.2001.18585

34. Silveira CRA, Menuchi MRTP, Simões CS, Caetano MJD, Gobbi LTB. Validade de construção em testes de equilíbrio: ordenação cronológica na apresentação das tarefas. Rev Bras Cineantropom Desempenho Hum. 2006;8(3):66-72. Doi: https://doi. org $/ 10.1590 / \% 25 x$ 